УДК 546.745:544.344

\title{
РАВНОВЕСИЕ НИКЕЛЯ И ТВЕРДЫХ РАСТВОРОВ ГАЛЛИЯ В НИКЕЛЕ С МОНООКСИДОМ УГЛЕРОДА
}

\author{
(C) 2017 А. Ю. Завражнов, А. В. Косяков \\ Воронежский государственный университет, Университетская пл., 1, 394018 Воронеж, Россия \\ e-mail:alzavr08@rambler.ru
}

Поступила в редакцию 28.12.2016 г.

\begin{abstract}
Аннотация. Целью настоящей работы являлось исследование селективного равновесия $\mathrm{Ni}^{(\text {тв })}+4 \mathrm{CO}^{\text {(газ) }} \mathrm{Ni}(\mathrm{CO})_{4}^{(\text {газ) }}$, направленное на получение термодинамических характеристик этого равновесия (константа равновесия, изменение энтальпии и энтропии в ходе реакции, коэффициент активности никеля в твердой фазе). В качестве источника никеля в такой обратимой реакции использовали измельченные в порошок металлы:чистый никельи твердые растворы галлия в никеле. Для исследования равновесия применяли спектрофотометрический метод, позволяющий получать электронные спектры поглощения паровой фазы в диапазоне длин волн 190-1100 нм и определять концентрации и парциальные давления каждой из молекулярных форм в паре. Для определения связи между абсорбционными свойствами карбонила никеля и концентрацией этого вещества в паре отдельно исследовали спектры поглощения пара $\mathrm{Ni}(\mathrm{CO})_{4}$, находящегося при низких температурах $\left(-70--25^{\circ} \mathrm{C}\right)$ в равновесии с твердым карбонилом никеля.

Получена температурная зависимость константы равновесия для реакции СО с фазой чистого никеля. Рассчитываемые по ней величины $K_{P}$ удовлетворительно коррелируют с литературными данными и оказываются средними между значениями, полученными в наиболее известных работах по данному равновесию. Величины $\Delta_{r} H_{298 \mathrm{~K}}^{\circ}$ и $\Delta_{r} S_{298 \mathrm{~K}}^{\circ}$, рассчитанные по II закону термодинамики для реакции с фазой чистого никеля, составляют $-168.12 \pm 3.97$ кДж/моль и $-460.27 \pm 10.23$ Дж/моль-К. Предложено объяснение очень большого рассогласования литературных данных по величинам $K_{P}, \Delta_{r} H$ и $\Delta_{r} S$.

При исследовании селективного равновесия между, твердыми растворами $\mathrm{Ga}_{x} \mathrm{Ni}_{1-x}$ (фаза на основе $\mathrm{Ni}$ ) и газообразными $\mathrm{CO}$ и $\mathrm{Ni}(\mathrm{CO})_{4}$ обнаружены аномально низкие величины коэффициента активности никеля в твердой фазе, которые могут указывать на чрезвычайно сильное химическое связывание между галлием и никелем в таком растворе.
\end{abstract}

Ключевые слова: карбонил никеля, гетерогенное равновесие, спектрофотометрическое исследование пара.

\section{ВВЕДЕНИЕ}

Карбонилы металлов являются важными прекурсорами при изготовлении пленочных гетероструктур с участием соединений металлов, входящих в состав этих комплексных соединений. Очень высокая ядовитость карбонилов, особенно, $\mathrm{Ni}(\mathrm{CO})_{4}$, делает актуальным синтез карбонилов «на месте» - с их немедленным расходованием в качестве прекурсоров. В случае карбонила никеля задача синтеза облегчается тем, что реакция (1) протекает с заметной скоростью даже при атмосферном давлении и использовании крупнозернистых порошков никеля.

$$
\mathrm{Ni}^{\prime \prime}+4 \mathrm{CO}^{\prime} \rightleftarrows \mathrm{Ni}(\mathrm{CO})_{4}{ }^{\prime}
$$

В равновесии (1) символ «" » означает компонент, находящийся в конденсированной фазе, символ «'»- в паровой фазе.

Заметим, что в качестве источника никеля в (1) может использоваться как чистое простое вещество, так и металлиды - твердые растворы и промежуточные фазы на основе никеля [1]. При этом реакция обладает высокой селективностью: при относительно низких давлениях, $\left(<10^{6}\right.$ Па) в нее не вовлекаются даже те металлы, которые сами способны образовывать карбонилы (Co, Fe) [2]. Вместе с тем, «прямой» синтез карбонила имеет низкую воспроизводимость выхода, что происходит по следующим причинам. Во-первых, достижение заметной концентрации $\mathrm{Ni}(\mathrm{CO})_{4}$ в паре в (1) ока- 
зывается возможным только в очень узком температурном интервале. Так для реакции с чистым неактивированным никелем при давлениях СО, близких к атмосферному, этот интервал составляет 70-130 ${ }^{\circ} \mathrm{C}$ : при $t \geq 130{ }^{\circ} \mathrm{C}$ равновесие почти нацело смещено влево, а при $t \leq 70{ }^{\circ} \mathrm{C}$ скорость реакции синтеза карбонила становится слишком мала. Вовторых, возможность получения карбонила по реакции (1) сильно зависит от природы и концентрации примесей в никеле - часто даже в тех случаях, когда эти концентрации малы. Так, растворение меди в твердом никеле - вплоть до концентрации 10 мол.\% $\mathrm{Cu}$ - лишь незначительно влияет на скорость (1) и на положение равновесия. Однако образование твердых растворов с участием алюминия даже при меньшем - по сравнению с медью - содержании в никеле делает синтез карбонила по реакции (1) практически невозможным [3].

Целью настоящей работы являлось получение термодинамических характеристик равновесия (1): константы равновесия, изменения энтальпии и энтропии в ходе реакции, величин коэффициентов активности никеля в твердой фазе. При этом главная задача исследования состояла в сравнении таких характеристик, получаемых для равновесия с участием чистого никеля, с аналогичными характеристиками при использовании в качестве конденсированной фазы твердых растворов галлия в никеле.

Выбор галлия в качестве второго компонента обусловлен тем, что именно в этой системе образуются одни из самых устойчивых интерметаллидов никеля (наиболее отрицательные значения $\Delta_{r} G_{298}^{0}$ в расчете на образование 1 моль фазы данной стехиометрии; еще более выгодным является образование промежуточных фаз в системе $\mathrm{Ni}-\mathrm{Al}$ однако фазы из последней системы в настоящем исследовании не использовали по причине высокой агрессивности алюминия и его сплавов к кварцевому стеклу) [4]. С учетом этого, изменение реакционной способности никеля при связывании с галлием должно проявляться наиболее отчетливо. Кроме того, ожидали, что исследование равновесия (1) с участием какой-либо широкогомогенной фазы системы Ni-Ga позволит сравнить величины химических потенциалов никеля (рассчитанные относительно чистого никеля), полученные экспериментально (см. [5]) с этими же величинами, вычисленными из уравнения Гиббса-Дюгема. Такая работа виделась возможной с учетом того, что концентрационные зависимости химического потенциала галлия на областях гомогенности интерме- таллидов системы Ni-Ga могут быть относительно легко получены при исследовании равновесия

$$
\begin{gathered}
2 \mathrm{Ga}_{\text {из фаз системы } \mathrm{Ga}-\mathrm{Ni}}^{\prime \prime}+\mathrm{GaHal}_{3}^{\prime} \rightleftarrows 3 \mathrm{GaHal}^{\prime} \\
(\mathrm{Hal}=\mathrm{Cl}, \mathrm{Br}, \mathrm{I}) .
\end{gathered}
$$

$T$ - $x$ диаграмма системы Ni-Ga характеризуется наличием 9 промежуточных фаз [6], для данной работы важна только фаза твердого раствора на основе никеля, область гомогенности которой простирается в сторону галлия до 10 мол.\% при комнатной температуре и до $20 \%$ вблизи $1000{ }^{\circ} \mathrm{C}$.

\section{МЕТОДИКА ЭКСПЕРИМЕНТА}

Исследование равновесия (1) проводили спектрофотометрически с использованием процедуры закаливания. Способ модификации оптической кюветы для такого эксперимента описан в [7]. В качестве конденсированной фазы в работе использовали порошки металлов: чистого никеля (Ni-00) и твердых растворов галлия в никеле с содержанием галлия 1 и 10 мол.\% с кристаллической структурой никеля (по данным выполненного нами РФА). Синтез твердых растворов проводили однотемпературным методом в печи индукционного нагрева при температуре $1500 \pm 200{ }^{\circ} \mathrm{C}$. Особенность конструкции печи состояла в том, что в процессе нагревания и сплавления гомогенизируемые навески элементов зависали внутри вакуумированных кварцевых ампул, не касаясь их стенок и не расплавляя сами ампулы, в которые эти навески были заключены. После синтеза кварцевые ампулы со сплавами помещали в муфельную печь, проводили отжиг при $1100{ }^{\circ} \mathrm{C}$ в течение 8 часов и охлаждение в режиме выключенной печи.

Все образцы (как никель, так и сплавы) измельчали в порошки с поперечным размером зерен 0.1-1.0 мм. Порошок металла помещали в оптическую кювету. Далее кювету вакуумировали, заполняли из кварцевого баллона газообразным монооксидом углерода до запланированной заранее величины давления и отпаивали. Порядок заполнения этой кюветы твердым веществом и требуемым газом при фиксированном давлении подробно описаны в [7]. Монооксид углерода получали термическим разложением безводного оксалата кальция при 400 С. Давление СО измеряли при помощи высокоточного цифрового манометра $X P_{i}-2$ с погрешностью измерения 26.6 Па. Давление монооксида углерода в различных экспериментах задавали от 0.3 до 0.7 атм. Верхняя граница диапазона давлений выбиралась таким образом, чтобы пар в 
системе оставался бы при комнатной температуре ненасыщенным даже при полном превращении СО в $\left[\mathrm{Ni}(\mathrm{CO})_{4}\right]$. Перед началом экспериментов отпаянную кювету помещали в муфельную печь и отжигали при $200{ }^{\circ} \mathrm{C}$ в течение 2 часов для снятия механических напряжений в приповерхностных слоях зерен никеля или его сплавов.

Равновесие, его закаливание и сканирование спектров. Для достижения равновесия (1) отпаянную кювету с веществами помещали в криотермостат, заполненный глицерином, задавали нужную температуру (от 80 до $130{ }^{\circ} \mathrm{C}$ ) и проводили непрерывный изотермический отжиг в течение нескольких десятков часов. Отжиг завершали закаливанием кюветы в дистиллированной воде, после чего при помощи спектрофотометра СФ-56 при комнатной температуре снимали спектр поглощения в диапазоне длин волн 190-360 нм. После этого описанные выше процедуры (отжиг, закаливание и сканирование спектра) повторяли и сравнивали результаты. Критерием установления равновесия при данной температуре была стабилизация положения спектральной кривой во времени в сочетании с возможностью количественного воспроизведения спектра при переходах как от большего содержания карбонила в паре (смещение равновесия влево), так и от меньшего (смещение вправо). После сканирования спектра, относящегося к равновесию при данной температуре, переходили к исследованию при другой температуре.

Определение молярных абсорбционныххарактеристик паров $\mathrm{Ni}(\mathrm{CO})_{4}$.

Важнейшим условием для использования спектрофотометрического метода является возможность определения величин парциальных давлений каждой из молекулярных форм равновесной паровой фазы. В случае равновесной смеси $\left(\mathrm{Ni}(\mathrm{CO})_{4}+\mathrm{CO}\right)$ хромофорной формой является именно карбонил никеля (высокая абсорбция на интервале длин волн 200-310 нм), а монооксид углерода практически не поглощает свет в «воздушном» УФ и видимом диапазонах спектра. Тогда молярная концентрация карбонила никеля напрямую находится из спектральных данных, если известен коэффициент мольной экстинкции карбонила $\varepsilon_{\lambda}$. Концентрация же СО в паре может быть вычислена, если известны температура и давление монооксида углерода в начале эксперимента, когда оптическая кювета заполнялась монооксидом углерода. В этом случае, а также для расчетов парциальных давлений $\mathrm{CO}$ и $\mathrm{Ni}(\mathrm{CO})_{4}$, использовали приближение идеального газа, которое считали корректным для используемой области температур и давлений.

$$
C_{\mathrm{CO}}^{\text {равн }}=\frac{p_{\mathrm{CO}}^{\text {исх }}}{R T_{0}}-4 C_{\mathrm{Ni}(\mathrm{CO})_{4}}^{\text {pas }},
$$

где в (3) $C_{\mathrm{CO}}^{\text {равн и }} C_{\mathrm{Ni}(\mathrm{CO})_{4}}^{\text {равн }}-$ равновесные концентрации оксида углерода (II) и карбонила никеля в паре, $p_{\mathrm{CO}}^{\text {исх }}$ - давление при заполнении кюветы угарным газом при температуре $T_{0}$.

Однако сложность в определении равновесной концентрации карбонила никеля заключалась в том, что данные по величине коэффициента мольной экстинкции карбонила никеля, извлекаемые из работ $[8,9]$, заметно различаются. Кроме того, данные по экстинкции пара $\mathrm{Ni}(\mathrm{CO})_{4}$, представленные рис. 1 и рис. 2 в работе [8], противоречат друг другу. По этой причине мы, дополнительно к основной работе, осуществляли градуировочный эксперимент по определению молярных абсорбционных характеристик паров карбонила никеля.

Для проведения такого градуировочного эксперимента исследовали спектры поглощения паров, находящихся в равновесии над твердым карбонилом никеля при температурах от -70 до $-21^{\circ} \mathrm{C}$. Сам карбонил никеля был получен по реакции (1) непосредственно в оптической кювете при проведении реакции в термостате при $+60{ }^{\circ} \mathrm{C}$ в течение 2 недель.

Для поддержания таких низких температур использовали описанную в [7] печь, входящую в состав спектрофотометрической установки и используемую в данной работе в качестве холодильника. Печь имела контур для жидкостного охлаждения, в которую подавали ток ацетона, охлажденного жидким азотом до температуры начала кристаллизации $\left(-95^{\circ} \mathrm{C}\right)$. Кроме того, для предварительного охлаждения установки термоизоляционный материал печи - легковесный кирпич - пропитывали жидким азотом.

После достижения минимальной температуры, необходимой для проведения эксперимента $\left(-70{ }^{\circ} \mathrm{C}\right)$, сканировали спектры поглощения пара $\mathrm{Ni}(\mathrm{CO})_{4}$, повышали температуру на несколько градусов $\left({ }^{\circ} \mathrm{C}\right)$, снова регистрировали спектр и снова с небольшим шагом повышали температуру. Последнее сканирование спектра проводили при температуре тройной точки карбонила никеля $\left(-21^{\circ} \mathrm{C},[10]\right)$. Для получения достаточного массива данных процедуру (вымораживания карбонила никеля с последующим нагреванием и получением спектров при разных температурах) повторяли еще 2 раза. 


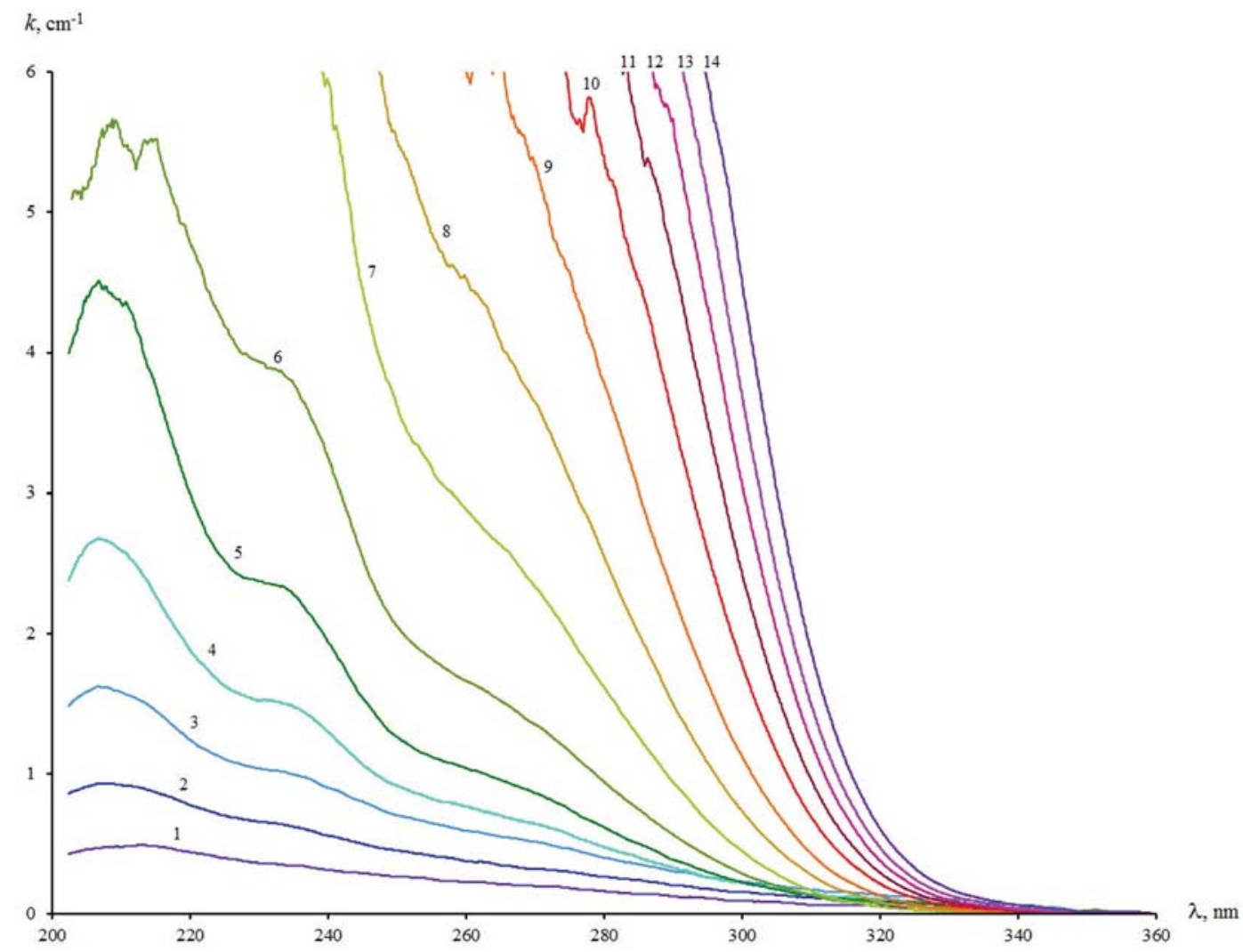

Рис. 1.Типичные спектры насыщенных паров карбонила никеля, полученные при разных температурах. Представленные кривые относятся к следующим температурам: 1 - -77, 2 - -70, 3 - -64, 4 - -58, 5 - -53, 6 - -47, $7--43,8--40,9--34,10--31,11--27,12--25,13--23,14--21{ }^{\circ} \mathrm{C}$

[Fig. 1. Representative spectrum of the saturated nickel carbonyl vapor at the following temperatures: $1--77,2--70$, $\left.3--64,4--58,5--53,6--47,7--43,8--40,9--34,10--31,11--27,12--25,13--23,14--21^{\circ} \mathrm{C}\right]$

Типичные спектры паров карбонила никеля, полученные при разных температурах, представлены на рис. 1.

Из этого рисунка видно, что заметное присутствие карбонила никеля в паре, равновесном с твердым кристаллическим карбонилом никеля, фиксируется уже при температурах $t \geq-70{ }^{\circ} \mathrm{C}$. Спектральная кривая имеет максимум при длине волны 206 нм и выраженное плечо при $\lambda=230.7$ нм.

Для определения мольного коэффициента экстинкции выполняли следующие процедуры. Для каждой температуры рассчитывали величину коэффициента оптического поглощения при длине волны $\lambda=230.7$ нм. С другой стороны, каждой такой температуре соответствовала определенная концентрация $C_{\mathrm{Ni}(\mathrm{CO})_{4}}$. Эту концентрацию находили с учетом уравнения для температурной зависимости насыщенного пара над твердым $\mathrm{Ni}(\mathrm{CO})_{4}$ [10] и закона идеального газа. В результате получали массив, состоящий из пар значений $\left(C_{\mathrm{Ni}(\mathrm{CO})_{4}}, k_{\lambda}\right)$. Этот массив обрабатывали в соответствии с линейной регрессией вида:

$$
C_{\mathrm{Ni}(\mathrm{CO})_{4}}=q_{230.7 \text { нм }} \cdot k_{230.7 \text { нм }},
$$

где $C_{\mathrm{Ni}(\mathrm{CO})_{4}}$ - концентрация карбонила в насыщенном паре, $k_{230.7 \text { нм }}-$ коэффициент оптического поглощения при указанной длине волны. Полученный при такой обработке коэффициент $q_{230.7 \text { нм }}$ позволял рассчитать мольный коэффициент экстинкции $\varepsilon_{\lambda}$ :

$$
\varepsilon_{230.7 \text { нм }}=\frac{1}{\left(q_{230.7 \mathrm{Hм}} \cdot \ln (10)\right)},
$$

Полученные результаты представлены на рис. 2 и сведены в табл. 1.

Рассчитанное с учетом приведенной в первой строке табл. 1 значения $q_{230.7 \text { нм }}$ величина мольной экстинкции $\varepsilon_{230.7 \text { нм }}$ составляет $5.59 \cdot 10^{6} \mathrm{~cm}^{2} /$ моль или $5590 \mathrm{M}^{-1} \mathrm{~cm}^{-1}$. Эта величина почти совпадает с данными [9] (5750 $\left.\mathrm{M}^{-1} \mathrm{~cm}^{-1}\right)$ и несколько отличается от результатов [8] (значения от 8160 до $13030 \mathrm{M}^{-1} \mathrm{~cm}^{-1}$ ). Рис. 2 показывает, что какая-либо заметная температурная зависимость коэффициента $q_{230.7 \text { нм }}$ и мольной экстинкции $\varepsilon_{230.7 \text { нм }}$ не прослеживается - в противном случае связь между $C_{\mathrm{Ni}(\mathrm{CO})_{4}}$ и $k_{230.7 \text { нм }}$ на рис. 2 была бы заметно нелинейной. 


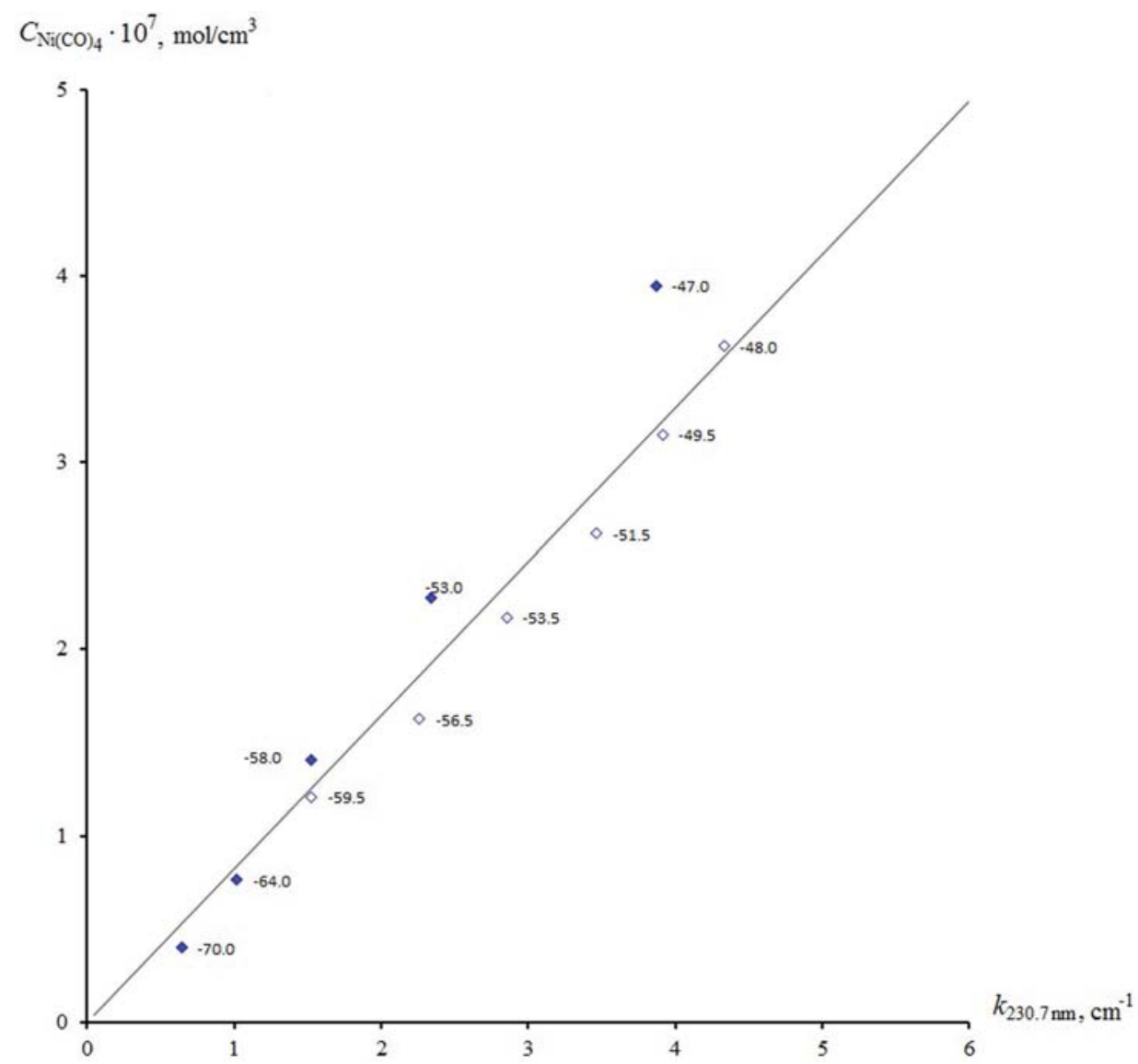

Рис. 2. Концентрационная зависимость коэффициента оптического поглощения карбонила никеля при длине волны 230.7 нм. Подписи у точек отвечают температурам равновесия кристаллического карбонила никеля с его насыщенным паром

[Fig. 2. The dependency of optical absorption coefficient (at wavelength of $230.7 \mathrm{~nm}$ ) on the gas phase concentration of $\mathrm{Ni}(\mathrm{CO})_{4}$. Point labels correspond the equilibrium temperatures]

Заметим, что корректное определение величины коэффициента оптического поглощения возможно, если эта величина оказывается в промежутке от 0.1 до 5-6 см ${ }^{-1}$. Однако по причине высокой мольной экстинкции карбонила никеля верхний предел $k_{230.7 ~ н м}$ достигается уже при концентрации карбонила никеля $4.2 \cdot 10^{-7}$ моль $/ \mathrm{cm}^{3}$, что соответствует давлению 1000 Па при комнатной температуре. В то же время, в эксперименте с исследованием температурной зависимости константы равновесия (1) ожидались гораздо большие концентрации.

По этой причине мы также рассчитывали площади под спектральными кривыми, ограниченными определенными значениями длин волн (в качестве характеристических определяли площади, ограниченные следующими длинами волн: 250-270, 270-290, 300-310 нм). При этом каждая величина площади соответствовала определенной концентрации карбонила никеля, которая задава- лась температурой. Полученные пары значений $\left(C_{\mathrm{Ni}_{(\mathrm{CO})}}, S_{\lambda_{1}, \lambda_{2}}\right)$, аналогично предыдущему случаю, обрабатывали в соответствии с линейной зависимостью:

$$
C_{\mathrm{Ni}(\mathrm{CO})_{4}}=Q_{\lambda_{1}, \lambda_{2}} \cdot S_{\lambda_{1}, \lambda_{2}}
$$

и находили коэффициент $Q_{\lambda_{1}, \lambda_{2}}$, который также является мольной характеристикой светопоглощения и по своему смыслу близок к величине, обратной мольному коэффициенту экстинкции. Полученные данные сведены в табл. 1.

Как следует из этой таблицы, использование для расчета концентрации $\mathrm{Ni}(\mathrm{CO})_{4}$ величин площадей под спектральной кривой, ограниченной длинами волн 300-310 нм, позволяет работать со значительно большими концентрациями этого вещества по сравнению с использованием значений коэффициента оптического поглощения при рекомендованной в [8] длине волны 230.7 нм $\left(k_{230.7 \text { нм }}\right)$. 
Таблица 1. Величины коэффициентов, связывающих абсорбционные свойства пара $\mathrm{Ni}(\mathrm{CO})_{4}$ (коэффициент оптического поглощения, площадь спектральной кривой) с концентрацией карбонила никеля в паре

[Table 1. Values for the constants in the dependences of $\mathrm{Ni}(\mathrm{CO})_{4}$ gas-phase optical absorption on the nickel carbonyl concentration (gas-phase)]

\begin{tabular}{|c|c|c|c|}
\hline $\begin{array}{c}\text { Обозначение величины } \\
\text { и размерность } \\
\text { [Designation and dimension } \\
\left.\text { of the constant }\left(\mathrm{mol} / \mathrm{cm}^{3}\right)\right]\end{array}$ & $\begin{array}{l}\text { Полученное } \\
\text { численное } \\
\text { значение } \\
\text { [Value] }\end{array}$ & $\begin{array}{c}\text { №№ уравнений } \\
\text { в тексте, которыми } \\
\text { эта величина вводится } \\
\text { [Numbers of the corre- } \\
\text { sponding equations in the } \\
\text { text] }\end{array}$ & $\begin{array}{c}\text { Верхний предел } \\
\text { концентраций (моль/см³), } \\
\text { ограничивающий использова- } \\
\text { ние данной величины } \\
\text { [The concentration upper limit } \\
\left.\left(\mathrm{mol} / \mathrm{cm}^{3}\right)\right]\end{array}$ \\
\hline$q_{230.7 \text { нм }}$, моль $/ \mathrm{cm}^{2}$ & $8.23 \cdot 10^{-7}$ & 4 & $4.2 \cdot 10^{-7}$ \\
\hline$Q_{250-270 \text { нм }}$, мОЛь $/ \mathrm{cm}^{3}$ & $1.02 \cdot 10^{-8}$ & 6 & $7.0 \cdot 10^{-7}$ \\
\hline$Q_{270-290 \text { нм }}$, моль $/ \mathrm{cm}^{3}$ & $1.77 \cdot 10^{-8}$ & 6 & $1,2 \cdot 10^{-6}$ \\
\hline$Q_{300-310 \text { нм }}$, моль $/ \mathrm{cm}^{3}$ & $1.00 \cdot 10^{-7}$ & 6 & $4.2 \cdot 10^{-6}$ \\
\hline
\end{tabular}

\section{РЕЗУЛЬТАТЫ ЭКСПЕРИМЕНТА}

По исследованию равновесия металлического никеля с монооксидом углерода было выполнено 2 эксперимента (отличались давлением вводимого исходно СО), результаты которых представлены в табл. 2. Для расчета величин концентраций и далее - парциальных давлений карбонила никеля в паре (как $p=C R T)$ - использовались данные для коэффициентов $q_{\lambda}$ и $Q_{\lambda_{1}, \lambda_{2}}$ (табл. 1) и абсорбционные характеристики $\left(k_{\lambda}, S_{\lambda_{1}, \lambda_{2}}\right)$, полученные при спектрофотометрических исследованиях при различных температурах, стартовых концентрациях оксида углерода (II) и составах твердой фазы на основе никеля (табл. 2).

Равновесные величины концентраций и давлений оксида углерода (II) определялись по материальному балансу по этому компоненту с учетом парциального давления исходно вводимого в кювету $\mathrm{CO}$, а также - свободного объема оптической кюветы и температуры (см. выражение 3). Полученные в этой работе данные температурной зависимости константы гетерогенного равновесия металлического никеля с СО представлены на рис. 3. На этот же рисунок нанесены аналогичные литературные данные. Обработкой экспериментальных данных $\ln \left(K_{P}\right)-1 / T$ по методу наименыших квадратов было получено выражение для температурной зависимости константы гетерогенного равновесия (1):

$$
\ln \left(K_{P}\right)=\frac{20221 \pm 478}{T}-(55.12 \pm 1.23) .
$$

Это уравнение коррелирует с аналогичными выражениями, указанными в работах [11] и [12], причем рассчитанные по нему величины $K_{P}$ оказываются меньшими, чем по результатам [11], большими, чем по [12] и наиболее хорошо согласовывались с данными [13].

Величины $\Delta_{r} H_{298 \mathrm{~K}}^{\circ}$ и $\Delta_{r} S_{298 \mathrm{~K}}^{\circ}$, рассчитанные по II закону термодинамики при использовании уравнения (7) и данных по теплоемкостям [14], составляют -

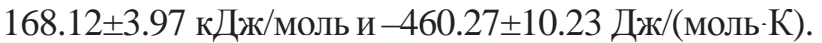
(О расчетах термодинамических величин при обработке данных экспериментов по II и III законам термодинамики подробнее см. [15], с. 31 и [16], с. 24.). Теплоемкость $C_{P}$ для каждого вещества находили для средней температуры 338 К; это значение использовали в расчетах как постоянную величину - такое упрощение сделано по причине очень различающихся между собой вариантов температурной зависимости $C_{P}$ в различных источниках. Увеличением погрешности за счет неточностей при определении теплоемкостей при оценке $\Delta_{r} H_{298 \mathrm{~K}}^{\circ}$ и $\Delta_{r} S_{298 \text { K }}^{\circ}$ пренебрегали. Рекомендуемые в настоящее время в интернет-справочниках термодинамические данные по карбонилу никеля в большинстве случаев цитируют работу [14], в которой величина $\Delta_{r} H_{298 \mathrm{~K}}^{\circ}$, составляет $-160.4 \pm 2.5$ кДж/моль (как среднее при обработке по II и III законам термодинамики), а для $\Delta_{r} S_{298 \mathrm{~K}}^{\circ}-417.27$ Дж/моль-К. Таким образом, результаты нашего исследования удовлетворительно согласуются с данными работы [13], хотя и отличаются от нее меньшими значениями, как энтальпии, так и энтропии реакции (1).

Перейдем к описанию результатов экспериментов с твердыми растворами Ga в Ni. Для такой твердой фазы селективное равновесие по-прежнему отражается уравнением (1), при этом для описания этого равновесия удобно использовать экспериментальную величину $K_{P}^{\#}$, которая связывает парциальные давления как 


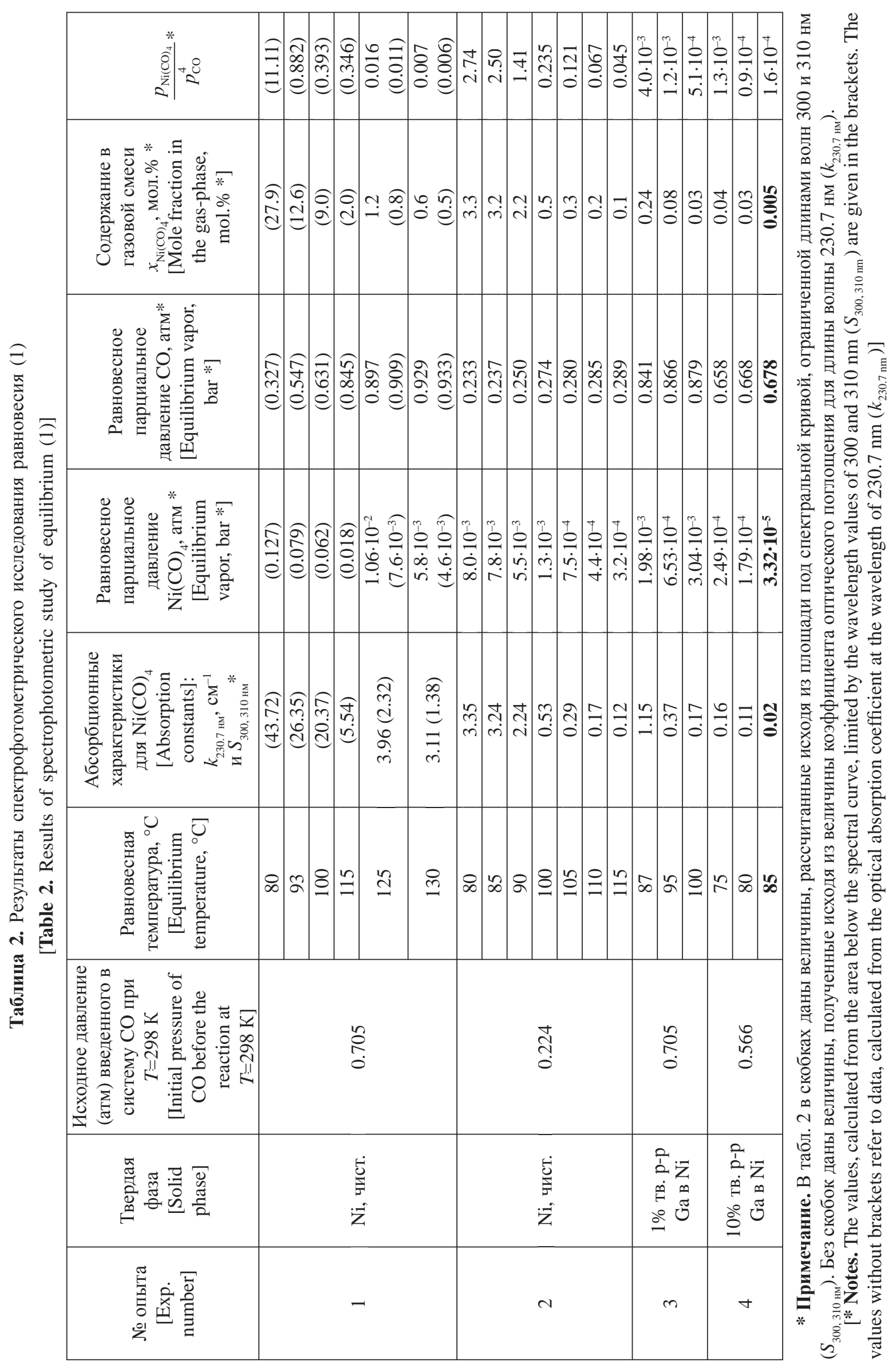




$$
K_{P}^{\#}=\frac{p_{\mathrm{Ni}(\mathrm{CO})_{4}}}{p_{\mathrm{CO}}^{4}}
$$

но при этом, в отличие от «истинной» константы равновесия $K_{P}$ является функцией состава конденсированной фазы, что видно из ее связи с химическим потенциалом никеля в сплаве (который сам, очевидно, зависит от состава):

$$
\mu_{\mathrm{Ni}}^{\text {в сплаве }}=R T \ln \left(K_{P}^{\#}\right)+\mu_{\mathrm{Ni}(\mathrm{CO})_{4}}^{\circ}-4 \mu_{\mathrm{CO}}^{\circ}
$$

где $\mu_{\mathrm{Ni}(\mathrm{CO})_{4}}^{\circ}-4 \mu_{\mathrm{CO}}^{\circ}-$ зависящая только от температуры разность стандартных потенциалов молекулярных форм газовой фазы. Поскольку химические потенциалы компонентов конденсированных фаз очень мало зависят от общего давления, то псевдоконстанты $K_{P}^{\#}$ полагали пренебрежимо мало зависящей от давления $P$. Для чистого никеля, очевидно $K_{P}^{\#}=K_{P}$. Таким образом, с учетом (9), на одном рисунке (рис. 3) можно отразить данные о химическом потенциале никеля в чистом простом веществе и его сплавах.

\section{ОБСУЖДЕНИЕ РЕЗУЛЬАТАТОВ}

Особенности равновесия (1) для случая, когда конденсированная фаза представлена твердыми растворами галлия в никеле. В экспериментах, в которых чистый никель заменяли твердым рас-

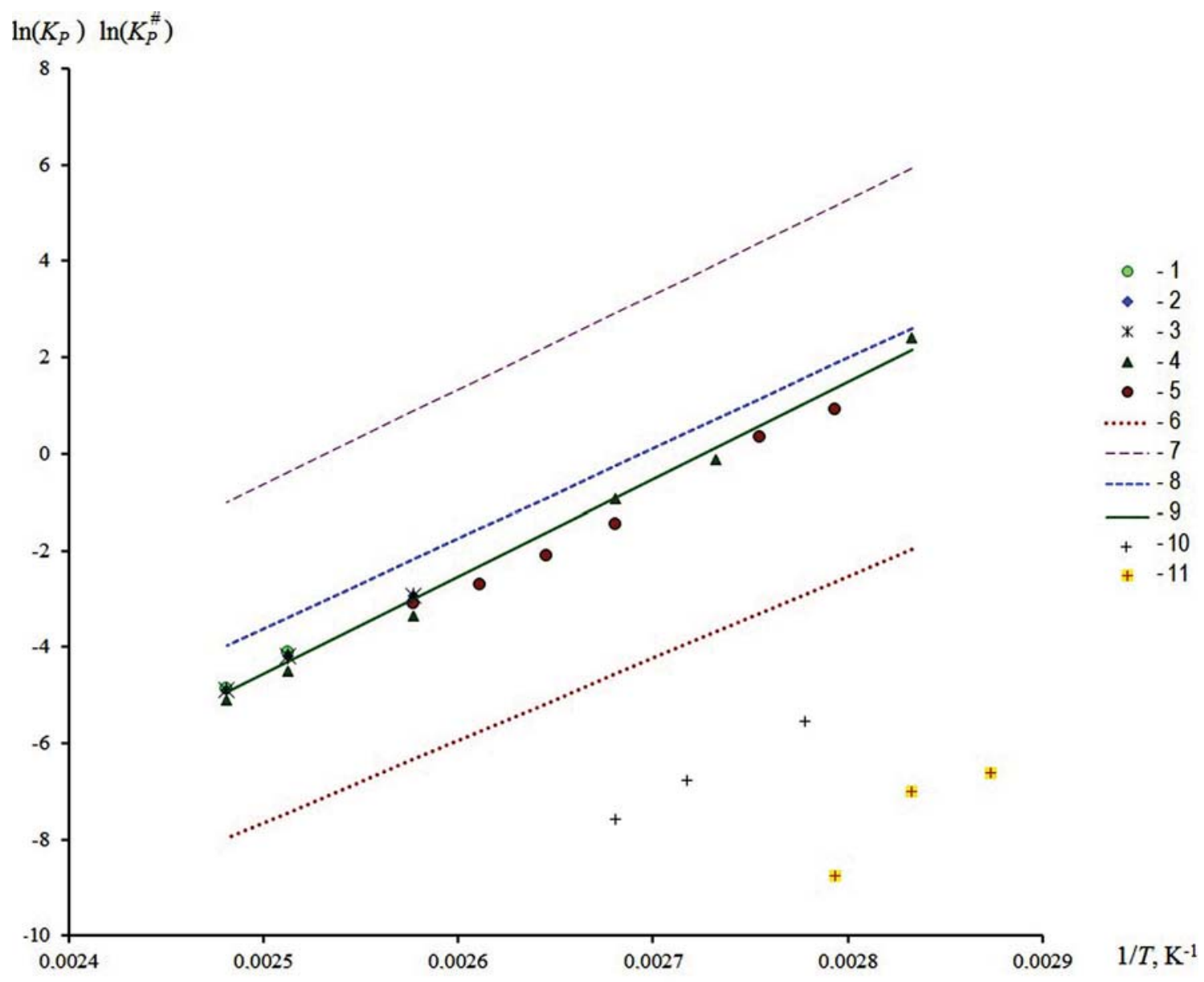

Рис. 3. Температурная зависимость константы гетерогенного равновесия $K_{P}$ (равновесия (1) с участием чистого никеля) или «псевдоконстанты» $K_{P}^{\#}$ (равновесия (1) с участием твердых растворов $\mathrm{Ga}_{x} \mathrm{Ni}_{1-x}$ ). Обозначения: $1-5-$ экспериментальные точки настоящей работы, 6-8 - литературные данные для равновесия с участием никеля: 6- [11], 7 - [12], 8 - [13], 9 - линеаризация по методу наименьших квадратов экспериментальных данных этой работы, относящихся к равновесиям с чистым никелем, 10, 11 - экспериментальные данные для равновесия с участием твердых растворов $\mathrm{Ga}_{x} \mathrm{Ni}_{1-x}: 10$ - сплав с содержанием $\mathrm{Ga} 1$ мол.\%, $11-10$ мол.\% Ga

[Fig. 3.The dependency of the heterogeneous constant $K_{P}$ (equilibrium reaction (1) for the pure metallic nickel) or the «pseudo-constant» $K_{P}^{\#}$ (equilibrium reaction (1) for the $\mathrm{Ga} \mathrm{Ni}_{x}$ solid solutions) on the reciprocal temperature. Notes: 1-5 - experimental points for equilibria with pure nickel (this work); 6-8 - data from the following sources: $6-$ [11], 7 - [12], 8 - [13]; 9 - least-square data of this work for the equilibria with pure nickel; 10, 11 - experimental points for equilibria with $\mathrm{Ga}_{x} \mathrm{Ni}_{1-x}$ solid solutions (this work): $\left.10-1 \mathrm{~mol} \% \mathrm{Ga}, 11-10 \mathrm{~mol} \% \mathrm{Ga}\right]$ 
твором галлия в никеле ( $x_{\mathrm{Ga}}=0.01$ и 0.10$)$, содержание карбонила никеля оказывалось значительно меньшим при прочих равных условиях (см. предпоследний столбец табл. 2.). Это хорошо видно по снижению величин $K_{P}^{\#}$ (а, значит, и $\mu_{\mathrm{Ni}}^{\text {велаве }) ~ п р и ~}$ введении галлия: при сравнении на рис. 3 положения прямой «9» (равновесие с чистым никелем) с расположением группы точек «10» $(1 \% \mathrm{Ga})$ и «11» $(10 \% \mathrm{Ga})$ такое снижение при $T=$ const составляет несколько порядков. Снижение активности никеля при сохранении кристаллической структуры никеля и при преобладающем содержании этого элемента в сплавах представлялось очень значительным и превосходило все ожидания. Для иллюстрации этого рассмотрим величину коэффициента активности никеля в сплавах $\left(\gamma_{\mathrm{Ni}}\right)$. Поскольку все исследуемые сплавы принадлежали одной фазе, представлялось возможным использовать выражение:

$$
K_{P}=\frac{p_{\mathrm{Ni}(\mathrm{CO})_{4}}}{p_{\mathrm{CO}}^{4} \cdot x_{\mathrm{Ni}} \cdot \gamma_{\mathrm{Ni}}}=\frac{K_{P}^{\#}}{x_{\mathrm{Ni}} \cdot \gamma_{\mathrm{Ni}}} .
$$

С учетом того, что для чистого никеля $K_{P}^{\#}=K_{P}$, для $\gamma_{\mathrm{Ni}}$ получаем:

$$
\gamma_{\mathrm{Ni}}=\frac{K_{P}^{\#}}{K_{P} \cdot x_{\mathrm{Ni}}},
$$

где в расчетах значения $K_{P}^{\#}$ подставляем как $\frac{p_{\mathrm{Ni}(\mathrm{CO})_{4}}}{p_{\mathrm{CO}}^{4}}$ из табл. (2) для сплавов, а $K_{P}-$ константа равновесия для чистого никеля.

Установлено, что для твердых растворов с содержанием галлия 1 мол.\% величина $\gamma_{\mathrm{Ni}}$ составляет (1.2-1.3) $10^{-3}$, при повышении концентрации галлия до 10 мол.\% эта величина уменьшается до $(0.45-1.10) \cdot 10^{-4}$. Если обнаруженный эффект действительно относится к состоянию истинного равновесия, то его можно считать чрезвычайно интересным. Дело в том, что в этом случае можно говорить об очень сильном взаимном химическом связывании растворенных галлия и никеля - причем таком, которое должно простираться за пределы первых координационных сфер растворенных атомов галлия.

Заметим, что столь малые величины коэффициента активности никеля могут быть кажущиими$c я$, т.е. такими величинами, которые не относятся к состоянию устойчивого равновесия между объемом твердой фазы и газом. Это может происходить из-за нарушения реакционной поверхности зёрен твердой фазы хемосорбцией или же по причине экранирования поверхности фазы твердого раствора индивидуальными промежуточными фазами системы $\mathrm{Ga}-\mathrm{Ni}$ с большим содержанием галлия (поскольку поверхность зерен обедняется никелем, удаляющимся в газ в виде карбонила). Действительно, низкие температуры исключают хоть сколько-нибудь заметную при реальных временах эксперимента диффузию никеля к поверхности зерен. В результате состояние термодинамического равновесия по отношению к объему сплавов может не достигаться. Однако для разбавленного (например, $1 \%$ ) твердого раствора эффект такого экранирования едва ли может быть существенным, поскольку в условиях эксперимента в пар переходит лишь очень небольшое количество никеля (менее $10^{17}$ атомов), что при развитой площади поверхности порошка соответствует удалению всего лишь считанных монослоёв никеля с поверхности зерен.

Таким образом, обнаруженный очень нетривиальный эффект нуждается в дальнейших исследований - возможно, с привлечением других равновесных селективных реакций.

Особенности равновесия (1) для случая, когда конденсированная фаза представлена чистым металлическим никелем. Вернемся к результатам, полученным для равновесия (1) с участием чистого никеля. На рис. 3 можно видеть огромный разброс температурных зависимостей констант гетерогенного равновесия, полученных разными авторами. Одна из причин такого рассогласования может состоять в том, что в ряде работ использовался активированный никель (полученный, например, пиролизом формиата $\left.\mathrm{Ni}(\mathrm{HCOO})_{2}[3]\right)$, что может давать завышенные значения константы $K_{P}$.

Другая причина, на наш взгляд, может быть связана с относительной близостью температурнастоящего эксперимента $\left(85-130{ }^{\circ} \mathrm{C}\right)$ температуре фазового перехода II рода в металлическом никеле. Этот переход связан с превращением «ферромагнетик - парамагнетик» с температурой Кюри $358{ }^{\circ} \mathrm{C}$ (для чистого $\mathrm{Ni}$ ). Общеизвестно, что фазовые переходы II рода характеризуются $\lambda$-образной зависимостью теплоемкости, а сама величина теплоемкости связана с химическими потенциалами компонентов фазы - т.е. - с реакционной способностью компонентов фазы (в термодинамическом смысле). Известно также, что температура Кюри для никеля [17] может заметно изменяться под влиянием даже малых концентраций примесей, что вызывает разный ход температурных зависимостей теплоемкости вблизи этой точки. Поскольку температурный интервал эксперимента лежит недалеко от точки Кюри (для чистого никеля заметное возрастание теплоемкости с температурой проис- 
ходит уже выше 100 $\mathrm{C}$ ) этот эффект может оказать существенное влияние на определяемую величину $K_{P}$, для которой могут фиксироваться большие рассогласования при работе с разными образцами, имеющими разный ход температурных зависимостей теплоемкости.

Использование в данной работе неактивированного никеля, достаточно высокая чистота образцов и процедура отжига порошка никеля перед началом эксперимента по исследованию равновесия (1) увеличивают, по нашему мнению, надежность получаемых данных по величинам константы гетерогенного равновесия. Однако для более надежного определения величин $\Delta_{r} H_{298 \mathrm{~K}}^{\circ}$ и $\Delta_{r} S_{298 \mathrm{~K}}^{\circ}$, требуется более подробный эксперимент, позволяющий получать большее количество экспериментальных пар $\left\{K_{P}, T\right\}$ и обрабатывать данные в соответствии с более сложными, чем (7) зависимостями, позволяющими учитывать изменения теплоёмкостных составляющих равновесия (1).

\section{ЗАКЛЮЧЕНИЕ}

В ходе спектрофотометрического исследования гетерогенного равновесия между монооксидом углерода и металлическим никелем или фазой твердого раствора $\mathrm{Ga} \mathrm{Ni}_{x}$ на основе никеля получены следующие наиболее важные результаты:

1. Показана возможность и перспективность исследования обсуждаемого равновесия при помощи спектрофотометрии паровой фазы. Для определения связи между абсорбционными свойствами карбонила никеля и концентрацией этого вещества в паре отдельно исследовали спектры поглощения пара $\mathrm{Ni}(\mathrm{CO})_{4}$, находящегося при низких температурах $\left(-70--25^{\circ} \mathrm{C}\right)$ в равновесии с твердым карбонилом никеля.

2. Получена температурная зависимость константы равновесия для реакции СО с фазой чистого никеля. Рассчитываемые по ней величины $K_{P}$ удовлетворительно коррелируют с литературными данными и оказываются средними между значениями, полученными в наиболее известных работах по данному равновесию. Величины $\Delta_{r} H_{298 \mathrm{~K}}^{\circ}$ и $\Delta_{r} S_{298 \mathrm{~K}}^{\circ}$, рассчитанные по II закону термодинамики для реакции с фазой чистого никеля, составляют $-168.12 \pm 3.97$ кДж/моль и

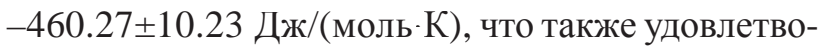
рительно согласуется с литературными данными. Предложено объяснение очень большого рассогласования литературных данных по величинам $K_{P}$, $\Delta_{r} H$ и $\Delta_{r} S$, основанное на большом влиянии даже малых количеств примесей на температуру Кюри для фазы никеля.

3. При исследовании селективного равновесия между, твердыми растворами $\mathrm{Ga} \mathrm{Ni}_{1-x}$ (фаза на основе $\mathrm{Ni})$ и газообразными $\mathrm{CO}$ и $\mathrm{Ni}(\mathrm{CO})_{4}$ обнаружены аномально низкие величины коэффициента активности никеля в твердой фазе, которые могут указывать на чрезвычайно сильное химическое связывание между галлием и никелем в таком растворе. Показана необходимость дальнейшей проверки этого факта в последующих работах.

\section{СПИСОК ЛИТЕРАТУРЫ}

1. Резник И. Д., Ермаков Г. П., Шнеерсон Я. М. Никель. Окисленные никелевые руды. Характеристика руд. Пирометаллургия и гидрометаллургия окисленных никелевых руд. М.: Наука и технологии, т. 2, 2004, $468 \mathrm{c.}$.

2. Joseph R. Davis. Nickel, Cobalt, and Their Alloys. ASM Specialty Handbook, Ohio Materials Park, USA, 2000, $442 \mathrm{p}$.

3. Козырев В.Ф. Дисс. ... докт. техн. наук «ОООГипроникель», Санкт-Петербург, 2007, 228 с.

4. Martosudirjo S., Pratt J- N. // Thermochimica Acta, 1976, vol. 17, pp. 183-194.

5. Завражнов А. Ю. Дисс. ... докт. хим. наук., Воронеж, 2004, 365 c.

6. Okamoto H. // J. of Phase Equilibria and Diffusion, Supplemental Literature Review: Section III, 2010, vol. 31, iss. 6, pp. 575-576.

7. Косяков А.В., Завражнов А.Ю., Наумов А.В. и др. // Вестник Воронежского государственного университета. Сер. Химия. Биология. Фармация. Воронеж, 2009, № 2, c. 28-39.

8. Pierloot K., Tsokos E., and Vanquickenborne L. G. // J. Phys. Chem. 1996, vol. 100, pp. 16545-16550.

9. Fuss W., Schmid W. E., and Trushin S. A. // J. Phys. Chem. A, 2001, vol. 105, pp. 333-339.

10. Brynestad J. Iron and Nickel Carbonyl Formation in Steel Pipes and its Preventions - Literature Survey. Oak Ridge National Laboratory Publishers, USA. 1976, $18 \mathrm{p}$.

11. Ross L. W., Haynie F. H., and Hochman R. F. // J. of Chemical and Engineering Data, 1964. vol. 9, № 3, pp. 339-340.

12. Fischer A. K., Cotton F. A., Wilkinson G.// Am. Chem. Soc., 1957, vol. 79, № 9, pp. 2044-2046.

13. Кипнис А.Я., Михайлова Н.Ф., Певзнер Г.Р. Карбонильный способ получения никеля. М.: Цветметинформация, 1972. 47 с.

14. Montei 1 Y., Raffin P., Bouix J. // Thermochimica Acta, 1988, vol. 125, pp. 327-346.

15. Киреев В. А. Методы практических расчетов в термодинамике химических реакций. М.: Химия, 1974, $536 \mathrm{c}$. 
16. Федоров В. А., Кузнецов Т. Н. Анализ и исследование полупроводниковых материалов. Самара: Изд-во Самар. гос. аэрокосм. ун-та, 2004, 84 с.
17. Бодряков В. Ю., Башкатов А. Н. // Заводская лаборатория. Диагностика материалов. 2004, т. 70 , №10,c. 41.

\title{
EQUILIBRIUM IN THE REACTION BETWEEN NICKEL OR GALLIUM-IN-NICKEL SOLID SOLUTIONS AND CARBON MONOOXIDE
}

\author{
(C) 2017 A. Yu. Zavrazhnov, A. V. Kosyakov \\ Voronezh State University, Universitetskaya sq., 1, 394018 Voronezh, Russia \\ e-mail:alzavr08@rambler.ru
}

Received 28.12.2016

\begin{abstract}
The purpose of this work was to investigate the heterogeneous equilibrium:

$$
\mathrm{Ni}_{(\mathrm{S})}+4 \mathrm{CO}_{(\mathrm{V})} \rightleftarrows \mathrm{Ni}(\mathrm{CO})_{4(\mathrm{~V})} \text { : }
$$

The data on the temperature dependence of this reaction equilibrium constant enables to measure important thermodynamic parameters (such as reaction enthalpy and entropy). Pure nickel or the solid solutions of gallium in nickel were used as equilibrium solid phases. It should be stressed that only nickel and nickel alloys form the stable carbonyl compound $\left(\mathrm{Ni}(\mathrm{CO})_{4}\right)$ in the reaction between solid metals and carbon monoxide at low vapor pressures (up to 1 bar). The considered reaction has high selectivity and in the case of nickel alloys only nickel (as a component) reacts with carbon monoxide.

High temperature spectrophotometry is the main method of vapor-pressure measurement in this work. Nickel carbonyl vapor has the specific bands in the near-UV absorption spectrum. The characteristic spectrum consists of a main peak at $206 \mathrm{~nm}$ and two shoulders at $230 \mathrm{~nm}$ and $270 \mathrm{~nm}$. The investigation of the optical absorption at these bands as a function of temperature allows one to determine the temperature dependence of nickel carbonyl concentration and, therefore, the equilibrium constant and some thermodynamic values for very reaction. To record the spectra during heating the experiment was carried out in an evacuated quartz cuvette. This cuvette was filled with nickel (nickel alloy) powder and with carbon monoxide gas. The vapor pressure was measured by the digital manometer (XP2i). Then the cuvette was sealed and annealed in the liquid thermostat for some days to reach an equilibrium state. After anneal the cuvette was quenched and analyzed for $\mathrm{Ni}(\mathrm{CO})_{4}$ concentration by spectral scanning. To find the dependency between the optical absorption coefficients $k_{\lambda}$ and the $\mathrm{Ni}(\mathrm{CO})_{4}$ concentration in the gas phase the separate experiments for the spectrum scanning of carbonyl saturated vapor $\left.\left(\mathrm{Ni}(\mathrm{CO})_{4(\mathrm{~S})} \rightleftarrows \mathrm{Ni}(\mathrm{CO})_{4(\mathrm{~V})} ; \mathrm{Ni(CO}\right)_{4(\mathrm{~L})} \rightleftarrows \mathrm{Ni}(\mathrm{CO})_{4(\mathrm{~V})}\right)$ were organized at very low temperatures $\left(-70--25^{\circ} \mathrm{C}\right)$.

Appreciable amounts of the nickel carbonyl were detected only at low temperatures $\left(<130^{\circ} \mathrm{C}\right)$, however at temperatures lower than $70^{\circ} \mathrm{C}$ the reaction rate was very low. For the reaction with pure nickel the calculated value of the reaction entropy is $-460.27 \pm 10.23 \mathrm{~J} / \mathrm{mol} \cdot \mathrm{K}$ and enthalpy is $-168.12 \pm 3.97 \mathrm{KJ} / \mathrm{mol}$. Concerning the solid solutions of gallium in nickel, the prompt decrease of the equilibrium gas-phase carbonyl concentration with the increase of gallium concentration in the contacting solid solution was found.
\end{abstract}

Keywords: vapor-pressure methods, spectrophotometry, heterogeneous equilibrium, vapor phase, absorption, nickel carbonyl.

\section{REFERENCES}

1. Reznik I. D. Ermakov G. P., Schneerson Ja. M. Nickel. Oxidized Nickel Ore. Characteristics of JOre. Pyrometallurgy and Hydrometallurgy of Oxidized Nickel Ores. Moscow, Science and Technology Publ., vol. 2, 2004, 468 p. (in Russian)
2. Joseph R. Davis. Nickel, Cobalt, and Their Alloys. ASM Specialty Handbook, Ohio Materials Park, USA, 2000, $442 \mathrm{p}$.

3. Kozyrev V. F. Diss. ... dr. Eng. Sci. «OOO Gipronickel», St. Petersburg, 2007, 228 p. (in Russian) 
4. Martosudirjo S., Pratt J-N. Thermochimica Acta, 1976, vol. 17, pp. 183-194. http://dx.doi.org/10.1016/00406031(76)85025-3

5. Zavrazhnov A. Ju. Diss. ... dr. Chem. Sci., Voronezh, 2004, 365 p. (in Russian)

6. Okamoto H. J. of Phase Equilibria and Diffusion, Supplemental Literature Review: Section III, 2010, vol. 31, iss. 6, pp. 575-576. DOI: 10.1007/s11669-010-9785-6

7. Kosyakov A.V., Zavrazhnov A. Ju., Naumov A. V., et al. Proceedings of Voronezh State University. Series: Chemistry. Biology. Pharmacy, Voronezh, 2009, no. 2, pp. 28-39. Available at: http://www.vestnik.vsu.ru/pdf/chembio/2009/02/2009-02-05.pdf (in Russian)

8. Pierloot K., Tsokos E., and Vanquickenborne L. G. J. Phys. Chem. 1996, vol. 100, pp. 16545-16550.

9. Fuss W., Schmid W. E., and Trushin S. A. J. Phys. Chem. A, 2001, vol. 105, pp. 333-339.

10. Brynestad J. Iron and Nickel Carbonyl Formation in Steel Pipes and its Preventions - Literature Survey. Oak Ridge National Laboratory Publishers, USA, 1976, $18 \mathrm{p}$.
11. Ross L. W., Haynie F. H., and Hochman R. F. J. of Chemical and Engineering Data, 1964. vol. 9, no. 3, pp. 339-340. DOI: 10.1021/je60022a007

12. Fischer A. K., Cotton F. A., Wilkinson G. Am. Chem. Soc., 1957, vol. 79, no. 9, pp. 2044-2046. DOI: 10.1021/ ja01566a005

13. Kipnis A. Ja., Mihajlova N. F., Pevzner G. R. A Carbonyl Method of Producing Nickel. Moscow, Tsvetmetinformatsiya Publ., 1972, 47 p. (in Russian)

14. Monteil Y., Raffin P., Bouix J. Thermochimica Acta, 1988, vol. 125, pp. 327-346.

15. Kireev V. A. Methods of Ppractical Calculations in the Thermodynamics of Chemical Reactions. Moscow, Chemistry Publ., 1974, 536 p. (in Russian)

16. Fedorov V. A., Kuznetsov T. N. Analysis and Research of Semiconductor Materials. Samara, Publishing House of Samar. State. Aerokosm. University Press, 2004, 84 p. (in Russian)

17. Bodryakov V. Y., BashkatovA. N. Inorganic Materials: English translation of selected articles from Zavodskaya Laboratoriya. Diagnostika Materialov, 2004, vol. 70, iss. 10 , p. 41.
Завражнов Александр Юрьевич - д. х. н., профессор кафедры общей и неорганической химии, Воронежский государственный университет; тел.: +7(915) 431126, e-mail: alzavr08@rambler.ru

Косяков Андрей Викторович - к. х. н., доцент кафедры общей и неорганической химии, Воронежский государственный университет; тел. +7(915) 5414596, e-mail: lavchukb@ mail.ru
Zavrazhnov Alexander Yu. - Dr. Sci. (Chem.), Professor, Department of General and Inorganic Chemistry, Voronezh State University; e-mail: alzavr08@ rambler.ru

Kosyakov Andrew V. - Cand. Sci. (Chem.), Assistant Professor, Department of General and Inorganic Chemistry, Voronezh State University; e-mail: lavchukb@mail.ru 